\title{
Achieving Optimal Backlog in Multi-processor Cup Games*
}

\author{
Michael A. Bender \\ Dept of CS, Stony Brook University \\ Stony Brook, NY, USA \\ bender@cs.stonybrook.edu
}

\author{
Martín Farach-Colton \\ Dept of CS, Rutgers University \\ New Brunswick, NJ, USA \\ martin@farach-colton.com
}

\author{
William Kuszmaul \\ CSAIL, MIT \\ Cambridge, MA, USA \\ kuszmaul@mit.edu
}

\begin{abstract}
Many problems in processor scheduling, deamortization, and buffer management can be modeled as single- and multi-processor cup games.

At the beginning of the single-processor $n$-cup game, all cups are empty. In each step of the game, a filler distributes $1-\varepsilon$ units of water among the cups, and then an emptier selects a cup and removes up to 1 unit of water from it. The goal of the emptier is to minimize the amount of water in the fullest cup, also known as the backlog. The greedy algorithm (i.e., empty from the fullest cup) is known to achieve backlog $O(\log n)$, and no deterministic algorithm can do better.

We show that the performance of the greedy algorithm can be exponentially improved with a small amount of randomization: After each step and for any $k \geq \Omega\left(\log \varepsilon^{-1}\right)$, the emptier achieves backlog at most $O(k)$ with probability at least $1-O\left(2^{-2^{k}}\right)$. We call our algorithm the smoothed greedy algorithm because if follows from a smoothed analysis of the (standard) greedy algorithm.

In each step of the $p$-processor $n$-cup game, the filler distributes $p(1-\varepsilon)$ unit of water among the cups, with no cup receiving more than $1-\delta$ units of water, and then the emptier selects $p$ cups and removes 1 unit of water from each. Proving nontrivial bounds on the backlog for the multi-processor cup game has remained open for decades. We present a simple analysis of the greedy algorithm for the multi-processor cup game, establishing a backlog of $O\left(\varepsilon^{-1} \log n\right)$, as long as $\delta>1 / \operatorname{poly}(n)$.

Turning to randomized algorithms, we find that the backlog drops to constant. Specifically, we show that if $\varepsilon$ and $\delta$ satisfy reasonable constraints, then there exists an algorithm that bounds the backlog after a given step by 3 with probability at least $1-$ $O\left(\exp \left(-\Omega\left(\varepsilon^{2} p\right)\right)\right.$.

We prove that our results are asymptotically optimal for constant $\varepsilon$, in the sense that no algorithms can achieve better bounds, up to constant factors in the backlog and in $p$. Moreover, we prove robustness results, demonstrating that our randomized algorithms continue to behave well even when placed in bad starting states.
\end{abstract}

\footnotetext{
*This work was supported in part by NSF Grants CCF 805476, CCF 822388, CNS 1408695, CNS 1755615, CCF 1439084, CCF 1725543, CSR 1763680, CCF 1716252, CCF 1617618, CCF 1314547, and CCF 1533644; and by an MIT Akamai Fellowship and a Fannie \& John Hertz Foundation Fellowship.

Permission to make digital or hard copies of all or part of this work for personal or classroom use is granted without fee provided that copies are not made or distributed for profit or commercial advantage and that copies bear this notice and the full citation on the first page. Copyrights for components of this work owned by others than the author(s) must be honored. Abstracting with credit is permitted. To copy otherwise, or republish, to post on servers or to redistribute to lists, requires prior specific permission and/or a fee. Request permissions from permissions@acm.org.

STOC '19, June 23-26, 2019, Phoenix, AZ, USA

(C) 2019 Copyright held by the owner/author(s). Publication rights licensed to ACM. ACM ISBN 978-1-4503-6705-9/19/06 . .\$15.00

https://doi.org/10.1145/3313276.3316342
}

\section{CCS CONCEPTS}

- Theory of computation $\rightarrow$ Scheduling algorithms; Design and analysis of algorithms; Online algorithms; Data structures design and analysis.

\section{KEYWORDS}

cup emptying, deamortization, discretized scheduling, parallelism, processor sharing, smoothed analysis

\section{ACM Reference Format:}

Michael A. Bender, Martín Farach-Colton, and William Kuszmaul. 2019. Achieving Optimal Backlog in Multi-processor Cup Games. In Proceedings of the 51st Annual ACM SIGACT Symposium on the Theory of Computing (STOC '19), fune 23-26, 2019, Phoenix, AZ, USA. ACM, New York, NY, USA, 10 pages. https://doi.org/10.1145/3313276.3316342

\section{INTRODUCTION}

A cup game $[1,12,15,17,18,27,33,34]$ is a multi-round game in which there there are $n$ cups, each initially empty, and two players. In each round, the filler distributes water to cups subject to some constraints, and then the emptier removes water from the cups subject to other constraints. For example, the filler may be constrained to add a total of at most one unit of water per round, while the emptier may be constrained to remove water from at most one cup.

The high-water mark after a given round is the maximum amount of water in any cup. The emptier's goal is to minimize the high-water mark and the filler acts as an adversary. In a cup game where the emptier uses a randomized strategy, the filler is oblivious; that is, the filler does not know the random choices made by the emptier (and thus does not know the state of the cups).

In this paper, we focus on two cup games that naturally arise in the study of processor scheduling $[1,18,27]$. These games (and their relaxations) have also appeared in a variety of other applications, ranging from deamortization [3, 4, 17-19, 24, 25, 32], to buffer management in network switches [5, 21, 23, 33], to quality of service in real-time scheduling $[1,8,27]$.

The single-processor cup game. Consider $n$ threads, where some amount of work arrives per round among the threads and the scheduler picks one thread to execute. The total volume of work that arrives per step is $1-\varepsilon$, where $\varepsilon$ is called the resource-augmentation parameter, and the total work scheduled is 1 . Rephrasing the problem as a cup game, at each step the filler may distribute up to $1-\varepsilon$ of water among all of the cups, and the emptier may select a cup and remove up to 1 units of water from it. In this context, the high-water mark is often referred to as the backlog.

The single-processor cup game and its variants have been studied extensively [1, 8, 17, 18, 29]. Notably, if the emptier follows the greedy algorithm and always removes water from the fullest cup, 
then the backlog does not exceed $O(\log n)$ [1]. In fact, this remains true even when $\varepsilon=0$. It is further known that no matter the value of $\varepsilon$, no deterministic algorithm can do better, even by an additive constant (see the variant of the lower bound presented in [12] and [18]). ${ }^{1}$

Our single-processor results. We show that the performance of the greedy emptying algorithm for the single-processor cup game can be exponentially improved with a small amount of randomization: After each step, and for any $k \geq \Omega(\log (1 / \varepsilon))$, the emptier achieves backlog at most $O(k)$ with probability at least $1-O\left(2^{-2^{k}}\right)$, and thus the backlog does not exceed $O(\log \log n)$ with high probability in $n$ (i.e., probability $1-1 / \operatorname{poly}(n))$ as long as $\varepsilon \geq \Omega(1 /$ polylog $n)$. Moreover, we show that these bounds are optimal, meaning that no algorithm can obtain the same probabilistic bounds for asymptotically smaller backlogs.

The algorithm, which we call the smoothed greedy algorithm, works as follows: The emptier begins the game by artificially adding to each cup $j$ a random quantity $r_{j} \in[0,1] .^{2}$ The emptier follows the deterministic greedy algorithm, except with a small but critical modification: If the fullest cup contains less than one unit of water, then the emptier does not remove any water at all.

This simple algorithm gives a smoothed-analysis explanation for why real-world systems should be expected to exhibit good behavior when following greedy strategies. Indeed, randomly perturbing the start-state of the cups is enough to give the deterministic algorithm the guarantees of the (optimal) randomized algorithm. In this sense, our randomized algorithm can be interpreted as a smoothed analysis of the standard greedy algorithm.

The small amount of resource augmentation used by the smoothed greedy algorithm makes it robust to the setting in which cups begin in a bad initial starting state. In particular, we show that if $b$ units of water are maliciously placed into cups at the beginning of the game, then for steps $t>\frac{b}{\varepsilon}$, the $b$ units of water have no affect on the guarantees given by the algorithm.

The multi-processor cup game. The version of the same scheduling question with $p>1$ processors has proved to be much harder to analyze $[1,8,27,29]$. The corresponding cup problem can be stated as follows. In each step of the multi-processor cup game, the filler distributes $(1-\varepsilon) p$ units of water among the cups with at most $1-\delta$ units of water in any given cup, where $\delta \geq 0$, and the emptier picks $p$ cups and removes a unit of water from each.

Note that if the filler is allowed to distribute water to cups arbitrarily, then the backlog is unbounded: the filler places more than one unit of water in cup 1 at each step, while the emptier can only remove a single unit from that cup. We include the restriction that $\delta \geq 0$ to exclude infeasible instances of the multi-processor cup game.

The difficulty of analyzing the multi-processor cup game stems from the restriction that the emptier remove at most one unit of

\footnotetext{
${ }^{1}$ The fact that the bounds are unaffected by the choice of $\varepsilon$ has led past authors to implicitly either take $\varepsilon$ to be zero $[1,8,29]$ or to be very large $[12,17,18]$. Combining the lower bound for the latter case with the upper bound for the former, one obtains the result for all $\varepsilon$ with no additional work.

${ }^{2}$ Many of our algorithms assume the ability to select random real thresholds in the range $[0,1)$. In $[10]$ we describe how to use low-precision thresholds in order to achieve the same guarantees, thereby reducing the number of random bits to $\Theta(\log n)$ per threshold.
}

water from each cup. This models the serial nature of each thread in the scheduling problem: distinct processors cannot make progress on the same thread simultaneously, even if that thread has a vastly greater backlog of work than its peers. As noted by Liu [29] and by later authors [8,27], this adds a "surprising amount of difficulty" to the scheduling problem. Providing provable backlog guarantees for the multi-processor cup game has been a long-standing open problem [1, 8, 27, 29].

Our multi-processor results. We prove that the greedy Emptyfrom-Fullest-Cups algorithm for the multi-processor cup game achieves a backlog of $O\left(\varepsilon^{-1} \log n\right)$ for any $0<\varepsilon<1$ and $\delta \geq \frac{1}{\operatorname{poly}(n)}$. When $\varepsilon$ is constant, we show that this is provably optimal up to constant factors.

Turning to randomized algorithms, we encounter an unexpected phenomenon: There exists a constant $c>0$ such that if $1 / 2 \geq \varepsilon \geq$ $p^{-1 / 3}$, and $2^{-c \varepsilon^{2} p}<\delta<1$, then there exists an algorithm that guarantees after each step a backlog of at most 3 with probability at least $1-2^{-\Omega\left(\varepsilon^{2} p\right)}$.

We further extend the guarantees of our randomized algorithm to bound the probability of larger backlogs. Namely, if $\delta \geq \frac{1}{\operatorname{poly}(p)}$, then after each step our algorithm achieves backlog $O(k / \varepsilon)$ with probability at least $1-O\left(e^{-e^{k}}\right)$. Additionally, in the case where $\varepsilon$ is constant, we prove lower bounds establishing that our probabilistic guarantees give optimal bounds, up to constant factors in the backlog $k$ and in $p$.

As in the single-processor case, we show that the smoothed greedy algorithm is robust in the sense that if it starts in a bad initial state, it quickly recovers to a low backlog. In particular, suppose $b$ units of water are maliciously placed into cups at the beginning of the game. Then the above guarantees for the multi-processor smoothed greedy algorithm hold after any step $t \geq 2 b / \delta$, except with an additional failure probability of $2^{-\Omega\left(t^{1 / 6}\right)}$.

A relaxed game for deamortization: the cup-emptying game. Cups games have been extensively used to analyze deamortization schemes of [3, 4, 17-19, 24, 25, 32]. In this setting, a relaxation of the single-processor cup game, which we call the cup-emptying game, is often used. In the cup-emptying game, the emptier is permitted to remove all of the water from a cup at each step, rather than just a single unit.

As in the single-processor cup game, a greedy emptier-who always empties from the fullest cup-can keep the backlog to $O(\log n)$ [17]. Using a more sophisticated randomized algorithm, the emptier can maintain the backlog at $O(\log \log n)$ with high probability in $n$ after any given round of the game [18].

Relationship with our results. Deitz et al. [18] pose finding a simpler algorithm as an open problem. Since the cup-emptying game is a relaxation of the single-processor cup game, our randomized algorithm provides a resolution to this open problem. Our algorithm additionally improves on the prior work by offering probability bounds of any backlog $k$, rather than applying only to $k=\log \log n$.

The cup-emptying game offers more power to the emptier than do the scheduling games. Consider, for example, the "multiprocessor" version of the cup-emptying game, in which the filler places $p$ units of water among cups and then the emptier flushes 
the water from $p$ cups. A solution to the "multi-processor" version follows trivially from the solution to the "single-processor" version, since the emptier can simply simulate the water poured by the filler as appearing in chunks of size one over the course of $p$ steps, and then perform the corresponding $p$ cup flushes (some of which may be to the same cup as each other). In contrast, the standard techniques for analyzing the single-processor cup game do not seem to generalize to the multi-processor case, and indeed the latter has remained open until now.

Other related work. Adler et al. [1], who proved the $O(\log n)$ bound on the backlog in the single-processor cup game, also considered a relaxation of the multi-processor cup game in which the $p$ units of water removed by the emptier need not be from distinct cups, establishing a tight bound of $\Theta(\log n)$ backlog for this problem (without the use of clairvoyance or resource augmentation). As noted by [8], however, this relaxed version of the problem can be reduced to the single-processor version of the same question, and is thus primarily interesting from the perspective of lower bounds rather than upper bounds.

Litman and Moran-Schein [27] proved a formal separation between the relaxed version and the non-relaxed versions, establishing that a deadline-based algorithm (which is completely clairvoyant) does provably worse in the multi-processor setting when it is not permitted to run the same thread on multiple processors concurrently. Litman and Moran-Schein [27] also showed that if the emptier in the multi-processor cup game is semi-clairvoyant, with knowledge of when the next $1+1 / \delta$ units of water arrive in each cup, then a simple deadline-based algorithm achieves backlog $O(1+1 / \delta)$. Providing provable guarantees for a fully non-clairvoyant emptier has, until now, remained open [1, 8, 27, 29].

In the context of packet-switching, Bar-Noy et al. [6] considered a variant of the single-processor cup game in which the filler is unconstrained as to how much water can be placed in the cups at each step, but must always place water in integer amounts. Rather than providing absolute bounds on backlog, which would be impossible, they show that the greedy algorithm achieves an $O(\log n)$ competitive ratio. Moreover, they show that no online algorithm, including randomized algorithms, can do better. Several of the same results were also discovered concurrently by Fleischer and Koga [20]. Subsequent work has considered weaker adversaries [16].

Extensive work has also focused on variants of the cup games in which the arrival rates are fixed [7-9, 22, 26, 28, 31]. That is, each cup $j$ receives the same amount $c_{j}$ at each step $t$. Baruah et al. [8] present a polynomial-time algorithm (per step of the game) that the emptier can follow in order to guarantee a maximum backlog of $O(1)$ in the single-processor cup game with fixed rates.

Some papers have explored variants of the multi-processor cup game in which the cups form the nodes of a graph, and the constraints on the emptier are a function of the graph structure [1215]. This setting models multiprocessor scheduling with conflicts between tasks $[14,15]$ and some problems in sensor radio networks [12].

Recently, cup-emptying games have been used to model memoryaccess heuristics in databases [11]. Here, whenever the emptier removes the water from a cup, the water is then redistributed according to some fixed probability distribution. The emptier's goal is ensure that the expected amount of water in the cup being emptied is large.

\section{A SMOOTHED ALGORITHM FOR THE SINGLE-PROCESSOR CUP GAME}

In the deterministic greedy algorithm for the single-processor cup game, the emptier removes water from the fullest cup at each step. It was proved by Adler et al. [1] that the greedy algorithm achieves backlog $O(\log n)$ at all times, independently of $\varepsilon \leq 1 / 2$, and the lower bound $[12,18]$ shows that no deterministic algorithm can do better, regardless of the choice of $\varepsilon$.

In this section, we show that randomization allows us to play this deterministic greedy game on a smaller effective set of cups, thus achieving an exponentially lower backlog.

Smoothed greedy algorithm. At the start of the game, the emptier selects random starting states $r_{1}, \ldots, r_{n} \in[0,1)$, and places $r_{j}$ water in each cup $j$. (This can, of course, be simulated rather than actually performed.) We refer to the randomization of the starting states as the smoothing. The emptier follows the deterministic greedy algorithm of emptying from the fullest cup with a small but critical modification: if the fullest cup contains less than one unit of water, then the emptier does not remove any water at all.

This modification ensures that the actions of the emptier do not affect the fractional amount of water (i.e., the amount of water modulo 1) in each cup, a property that plays a critical role in the analysis.

The smoothing, which is only performed once at the start, results in stronger bounds on the backlog, as follows:

Theorem 1. For all $\varepsilon \leq 1 / 2, t \in \mathbb{N}$, and $k \geq 3 \log \frac{1}{\varepsilon}$, the probability that the smoothed greedy algorithm has backlog at least $k$ after step $t$ is $(1 / 2)^{2^{\Omega(k)}}$.

Corollary 1. For $\varepsilon=\Omega(1 / \operatorname{polylog}(n))$ and every step $t$, the backlog is $O(\log \log n)$ at step $t$ with high probability in $n$.

As we will see, the smoothed greedy algorithm also maintains the bound of the deterministic greedy algorithm, deterministically guaranteeing that the backlog never exceeds $O(\log n)$.

In order to prove Theorem 1, we define a cup game where the number of cups can change dynamically: arbitrarily many new empty cups may be added by the filler at the beginning of each step, and empty cups are removed at the end of a step. We call this the dynamic single-processor cup game and prove:

Lemma 1. Suppose the emptier follows the greedy algorithm in the dynamic single-processor cup game, and let $n_{t}$ be the number of cups present after the $t$-th step in the game. Then, for any $\varepsilon$, the fullest cup after the $t$-th step contains $O\left(\log n_{t}\right)$ water.

Proof. To analyze the dynamic cup game $G$, we consider instead the dynamic cup game $G^{\prime}$ occurring on the cups in $G$ containing more than one unit of water. The fill of each cup in $G^{\prime}$ is one smaller than the fill of the corresponding cup in $G$. We wish to show that the backlog after the $t$-th step in game $G^{\prime}$ is at most $O\left(\log n_{t}\right)$, the number of cups in $G$. The key insight is that, whenever a cup is removed from $G$, the game $G^{\prime}$ must contain no cups (since no cups in $G$ can contain more than one unit of water). Thus we can 
think of the game $G^{\prime}$ as restarting whenever the game $G$ has a cup removed. On the other hand, if we consider a sequence of steps $t_{1}+1, \ldots, t_{2}$ in which $G^{\prime}$ initially contains no cups, and in which the number of cups in $G$ gradually increases from $n_{t_{1}}$ to $n_{t_{2}}$, then $G^{\prime}$ never contains more than $n_{t_{2}}$ cups during that sequence. Thus $G^{\prime}$ has backlog trivially bounded by $O\left(\log n_{t_{2}}\right)$ after step $t_{2}$ using the standard bound for the greedy algorithm on $n_{t_{2}}$ cups.

The smoothed greedy algorithm can be viewed as playing an instance of the dynamic single-processor cup game on the active cups, where we define a cup to be active if the cup contains more than one unit of water, and inactive otherwise. In the dynamic game, each active cup is taken to contain one fewer units of water than the cup actually contains, and the inactive cups are considered to be empty and removed from the game. A cup is added to the dynamic game when the filler makes it active. Bounding the number of active cups by $2^{k}$ will then yield a backlog of $O(k)$.

The difficulty in bounding the number of active cups directly is that there may be a series of time steps during which the emptier removes water primarily from cups containing more than two units of water, thereby failing to make those active cups inactive, but during which the filler manages to increase the number of active cups significantly.

Rather than bounding the number of active cups directly, we instead bound a different quantity, which we call the integer fill. Define $f_{j}(t)$ to be the amount of water in cup $j$ after step $t$. The integer fill after step $t$ is $\sum_{j=1}^{n}\left\lfloor f_{j}(t)\right\rfloor$, that is, the number of integer thresholds crossed within the cups. The integer fill upper bounds the number of active cups, since each active cup contributes at least 1 to the integer fill.

The integer fill is more tractable to bound than the number of active cups because, at each step, the emptier reduces the integer fill by one, unless the integer fill is already zero. This property ensures that, in order for the integer fill to exceed $2^{k}$ after a step $t$, the following must be true: There must be some $\ell>0$ so that in the $\ell$ steps leading up to $t$ (inclusive), at least $2^{k}+\ell$ integer thresholds were crossed by the filler ${ }^{3}$. We call any $\ell$ satisfying the latter property a $2^{k}$-backlog witness.

To bound the probability that there exists a backlog witness $\ell>0$, we first bound the probability that any fixed cup crosses an integer threshold during a given step. Define $c_{j}(t)$ to be the amount of water inserted into cup $j$ by the filler during a step $t$. Recall that in the smoothed greedy algorithm, the emptier always removes water in integer quantities. Thus, the amount of water modulo 1 in a cup $j$ after step $t$ is

$$
r_{j}+\sum_{i=1}^{t} c_{j}(i)(\bmod 1),
$$

regardless of the actions of the emptier. From Eq. 1 we see that, although the random quantity $r_{j}$ of water is inserted into the cup $j$ only once at the start of the game, it continues to randomize the amount of water modulo 1 at each step $t$. This yields the following lemma:

\footnotetext{
${ }^{3}$ More specifically, this property must be satisfied by the $\ell$ such that $t-\ell$ is the most recent step to have ended with integer fill zero.
}

Lemma 1. The probability that cup j crosses an integer threshold in step $t$ is exactly $c_{j}(t)$, the amount of water placed into the cup. Moreover when one cup crosses thresholds is independent of when other cups do.

Proof. By Eq. 1, an integer threshold is crossed in cup $j$ if and only if $r_{j}+\sum_{i=1}^{t} c_{j}(i)(\bmod 1) \in\left[0, c_{j}(t)\right)$. Since the quantity on the left side is uniformly distributed in $[0,1)$, the probability of a threshold crossing is exactly $c_{j}(t)$.

By Lemma 1, the expected number of thresholds crossed during any given step $t$ is $\sum_{j} c_{j}(t) \leq 1-\varepsilon$.

Integer-threshold crossings are not independent between steps, however. For example, if the filler places $1 / 2$ a unit of water in cup $j$ during a step, and then another $1 / 2$ a unit of water in cup $j$ during the next step, then exactly one of the two steps will cross an integer threshold in cup $j$.

Nonetheless, we can analyze $t$ consecutive steps together and express the number of integer thresholds crossed as a sum of independent indicator random variables. Define $\bar{x}=x-\lfloor x\rfloor$ to be the fractional part of $x$ for any non-negative real number $x$. If $u_{j}$ water is placed in cup $j$ during the $t$ steps, then the cup contributes $\left\lfloor u_{j}\right\rfloor$ indicator random variables that are deterministically set to 1 , and one indicator random variable whose value is 1 with probability $\overline{u_{j}}$. This is captured formally in Lemma 2.

Lemma 2. Consider a sequence of $t$ consecutive steps in the cup game in which the emptier follows the smoothed greedy algorithm. Then the number of integer thresholds crossed during the $t$ steps can be expressed as a sum of independent zero-one random variables with total mean at most $(1-\varepsilon) t$.

Proof. Let $\ell, \ell+1, \ldots, \ell+t-1$ be the indices of the steps being considered. For any cup $j$, let $a_{j}$ denote the total amount of water placed in the cup by the filler prior to the $\ell$-th step, and let $b_{j}$ denote the amount of the water placed in the cup during steps $\ell, \ldots, \ell+t-1$. That is, $a_{j}=\sum_{s<\ell} c_{j}(\ell)$ and $b_{j}=\sum_{\ell \leq s<\ell+t} c_{j}(\ell)$. Since $r_{j}$ units of water are poured into each cup $j$ at the beginning of the game, the total amount of water placed in the cup prior to step $\ell$ is $r_{j}+a_{j}$, and the total amount of water placed in the cup prior to step $\ell+t$ is $r_{j}+a_{j}+b_{j}$. The emptier only ever removes integer amounts from a cup, meaning that the number of integer thresholds crossed in the steps $\ell, \ldots, \ell+t-1$ is independent of which steps the emptier targeted cup $j$ in. Indeed, the precise number of integer thresholds crossed in cup $j$ is given by

$$
\left\lfloor r_{j}+a_{j}+b_{j}\right\rfloor-\left\lfloor r_{j}+a_{j}\right\rfloor .
$$

The first $\left\lfloor b_{j}\right\rfloor$ units of water poured into the cup during the steps $\ell, \ell+1, \ldots, \ell+t-1$ will cross $\left\lfloor b_{j}\right\rfloor$ integer thresholds in total. The final $\overline{b_{j}}$ units of water poured will cross an integer threshold if and only if

$$
\left\lfloor r_{j}+a_{j}+\overline{b_{j}}\right\rfloor>\left\lfloor r_{j}+a_{j}\right\rfloor .
$$

This, in turn, occurs if and only if $\overline{r_{j}+a_{j}} \in\left[1-\overline{b_{j}}, 1\right)$. Because $\overline{r_{j}+a_{j}}$ is uniformly random (based on $r_{j}$ ), the probability of an extra threshold crossing is $\overline{a_{j}}$. Therefore, the number of integer 
thresholds crossed in cup $j$ can be written as a sum of indicator variables

$$
X_{1}+X_{2}+\cdots+X_{\left\lfloor b_{j}\right\rfloor}+Y,
$$

where each $X_{i}$ takes value 1 with probability one, and where $Y$ is an indicator variable taking value 1 with probability $\overline{a_{j}}$, depending on $r_{j}$. Noting that the $r_{j}$ 's are independent between cups completes the proof.

Applying Lemma 2, we can bound the probability that there exists a backlog witness $\ell>0$.

Lemma 3. For any $m \geq 1 / \varepsilon^{3}$, the probability that the integer fill after step $t$ is $m$ or greater is at most $2^{-\Omega\left(m^{1 / 3}\right)}$.

Proof. Let $S_{\ell}$ denote the number of integer thresholds crossed in steps $t-\ell+1, \ldots, t$. Recall that the integer fill after a step is $m$ or greater only if there exists a $m$-backlog witness, meaning that $S_{\ell} \geq \ell+m$ for some $\ell$. By Lemma 2, $S_{\ell}$ can be expressed as a sum of independent indicator variables, and $\mathbb{E}\left[S_{\ell}\right]=(1-\varepsilon) \ell$.

When $\ell \leq m$, the probability that $S_{\ell} \geq \ell+m$ is exponentially small in $m$ :

$$
\operatorname{Pr}\left[S_{\ell} \geq \ell+m\right] \leq \operatorname{Pr}\left[S_{\ell} \geq \mathbb{E}\left[S_{\ell}\right]+m\right] \leq e^{-m / 3},
$$

where the final inequality uses a Chernoff bound. By a union bound, the probability that any $\ell \leq m$ is a $m$-backlog witness is at most $m e^{-m / 3} \leq 2^{-\Omega(m)}$.

On the other hand, when $\ell>m$, the probability that $S_{\ell} \geq \ell+m$ is exponentially small in $\varepsilon^{2} \ell$ :

$\operatorname{Pr}\left[S_{\ell} \geq \ell+m\right] \leq \operatorname{Pr}\left[S_{\ell} \geq \mathbb{E}\left[S_{\ell}\right]+\varepsilon \ell\right] \leq e^{-\varepsilon^{2} \mathbb{E}\left[S_{\ell}\right] / 3} \leq e^{-\varepsilon^{2} \ell / 6}$, where the second to final inequality uses a Chernoff bound.

Summing over all $\ell>m$ yields a geometric series. Specifically,

$$
\begin{aligned}
\operatorname{Pr}\left[S_{\ell} \geq \ell+m \text { for some } \ell>m\right] & \leq \sum_{\ell>m} \operatorname{Pr}\left[S_{\ell} \geq \ell+m\right] \\
& \leq \sum_{\ell>m} e^{-\varepsilon^{2} \ell / 6} \\
& =e^{-\varepsilon^{2} m / 6} \sum_{\ell>0}\left(e^{-\varepsilon^{2} / 6}\right)^{\ell} \\
& <e^{-\varepsilon^{2} m / 6} \frac{1}{1-e^{-\varepsilon^{2} / 6}} \\
& \leq e^{-\varepsilon^{2} m / 6} O\left(1 / \varepsilon^{2}\right) .
\end{aligned}
$$

Assuming $m \geq 1 / \varepsilon^{3}$, this becomes $e^{-\Omega\left(m^{1 / 3}\right)}$.

Combining the cases of $m \leq \ell$ and $m>\ell$, the probability that there exists a $m$-witness is at most $e^{-\Omega(m)}+e^{-\Omega\left(m^{1 / 3}\right)} \leq e^{-\Omega\left(m^{-1 / 3}\right)}$, as desired.

Putting the pieces together, we can complete the proof of Theorem 1.

Proof of Theorem 1. Consider $k$ such that $3 \log \frac{1}{\varepsilon} \leq k$, meaning that $1 / \varepsilon^{3} \leq 2^{k}$. By Lemma 3 , with probability at least $1-$ $e^{-\Omega\left(2^{k / 3}\right)}$, the integer fill after step $i$ is at most $2^{k}$. Since the integer fill is at least as large as the number of active cups, it follows that there are at most $2^{k}$ active cups. Applying Lemma 1 to the active cups, we get that the backlog is at most $O(k)$, as desired.

\section{DETERMINISTIC RESULTS FOR THE MULTI-PROCESSOR CUP GAME}

Recall that the multi-processor cup game works as follows. At each step the filler is allowed to distribute up to $(1-\varepsilon) p$ units of water among the cups, placing up to $1-\delta$ units in any individual cup; and then the emptier is permitted to remove up to one unit of water from each of up to $p$ distinct cups. As in the single-processor cup game, the emptier's goal is to minimize backlog.

In this section we analyze the (deterministic) greedy algorithm for the multi-processor cup game, in which the emptier's strategy is to simply always remove (up to) one unit of water from the $p$ fullest cups. We show the following:

Theorem 2. For $\varepsilon \in(0,1)$ and $\delta \geq \frac{1}{\operatorname{poly}(n)}$, the greedy algorithm achieves maximum backlog $O\left(\frac{1}{\varepsilon} \log n\right)$ on a multi-processor cup game with $n$ cups.

To introduce the approach for proving Theorem 2, we begin by revisiting the single-processor cup-emptying game [18].

\subsection{Revisiting the Cup-Emptying Game}

Recall that in each step of the cup-emptying game, the filler distributes one unit of water among the cups, and then the emptier removes all of the water from some cup. A result that has found numerous applications in deamortization is that, if the emptier follows the greedy algorithm, then the backlog is $O(\log n)[3,4,17-$ $19,24,25,32]$. In this subsection, we present a new proof of the result. In additional to bringing new intuition to the problem, our proof also has the property that it can easily be adapted to work even if the emptier does not remove from the fullest cup at each step but instead removes from a cup whose fill is within $O(1)$ of the maximum.

Recall that $f_{j}(t)$ denotes the amount of water in cup $j$ after step $t$. The key insight in the proof is to examine the potential function,

$$
\phi(t)=\sum_{j=1}^{n}(1.1)^{f_{j}(t)} .
$$

We will show that $\phi(t)$ does not exceed $O(n)$ by proving that at the end of each step $t$, either $\phi(t)<\phi(t-1)$, or no cup contains more than 2 units of water (and thus $\phi(t) \leq O(n)$ ). The bound on $\phi(t)$ prevents any single cup from ever having fill greater than $O(\log n)$.

To simplify the proof, we actually use a slightly different potential function,

$$
\phi(t)=\sum_{j=1}^{n} \int_{x=0}^{f_{j}(t)} 1.1^{\lceil x\rceil} d x .
$$

Using this potential function, the proof proceeds as follows. Consider a given step $i$. Let $\ell$ be the fill of the fullest cup after the filler takes their turn. Assume that $\ell \geq 2$; otherwise no cup contains more than 2 units of water, in which case $\phi(t)=O(n)$. The water added to the cups increases the potential by at most $1.1^{\lceil\ell\rceil}$ over $\phi(t-1)$. On the other hand, the emptier removes during step $t$ at least 2 units of water from the fullest cup, thereby decreasing the potential by at least

$$
1.1^{\lfloor\ell\rfloor}+1.1^{\lfloor\ell\rfloor-1} \geq 1.1^{\lceil\ell\rceil} .
$$

Thus the potential after step $t$ satisfies $\phi(t) \leq \phi(t-1)$. 
Since either $\phi(t)=O(n)$ or $\phi(t) \leq \phi(t-1)$ for each $t>0$, and since $\phi(0)$ is trivially 0 , the potential $\phi(t)=O(n)$ for all $t \geq 0$. This, in turn, implies that $f_{j}(t) \leq O(\log n)$ for all cups $j$ and steps $t$.

\subsection{Proving Theorem 2}

The proof of Theorem 2 generalizes the ideas from the previous section.

Proof of Theorem 2. Define the potential function,

$$
\phi(t)=\sum_{j=1}^{n} \int_{x=0}^{f_{j}(t)}(1+\varepsilon)^{\lceil x\rceil} d x .
$$

We prove that $\phi(t) \leq O(\operatorname{poly}(n))$ for all $t$. This, in turn, bounds each $f_{t}(j)$ to be at most $O\left(\log _{1+\varepsilon} \operatorname{poly}(n)\right)=O\left(\frac{1}{\varepsilon} \log n\right)$.

For a given step $t$, we consider two cases separately:

Case 1: The emptier removes a full unit of water from each of $\boldsymbol{p}$ cups during step $\boldsymbol{t}$. In this case, we break the emptying in step $t$ into three substeps, beginning after the filler has taken their turn: (1) The emptier identifies the $p$ fullest cups $D=\left\{d_{1}, \ldots, d_{p}\right\}$; (2) The emptier removes from each of the cups $d_{i}$ any water that was poured into it during step $t$; (3) The emptier removes additional water so that a total of one unit has been removed from each of the cups $d_{1}, \ldots, d_{p}$. Note that the assumption of Case 1 guarantee that substep (3) is well defined.

Let $h$ denote the fill of the emptiest cup in the set $\left\{d_{1}, \ldots, d_{p}\right\}$ at substep (1). Let $\ell$ be amount of water that the filler places in cups outside of $D$, which thus remain in the system after substep (2). The total contribution of the $\ell$ units of water to the potential can be at most $\ell(1+\varepsilon)^{\lceil h\rceil}$.

The amount of water removed in substep (2) is at most $p \cdot(1-$ $\varepsilon)-\ell=p-(\ell+\varepsilon p)$. Thus at least $\ell+\varepsilon p$ further units of water are removed during substep (3). The removal of water that occurs in substep (3) decreases the potential by at least

$$
(\ell+\varepsilon p)(1+\varepsilon)^{\lceil h\rceil-1} \geq(1+\varepsilon) \cdot \ell \cdot(1+\varepsilon)^{\lceil h\rceil-1} \geq \ell \cdot(1+\varepsilon)^{\lceil h\rceil} .
$$

Thus the potential $\phi(t)$ at the end of step $t$ satisfies $\phi(t) \leq \phi(t-1)$. Case 2: At no point during step $t$ do $p$ or more cups contain one or more units of water. Call the cups that contain more than one unit of water at the end of the step the heavy hitters. Any water placed in non-heavy hitters during step $t$ can increase the potential by at most $2 n$ in total. On the other hand, any water that is placed in a heavy hitter during step $t$ is then immediately removed by the emptier. Additionally, the emptier removes from each heavy hitter at least $\delta$ units of water that were present at the beginning of the step. If the fullest heavy hitter has fill $\ell$ at the beginning of the step, then this removal will reduce the potential by at least

$$
(1+\varepsilon)^{\lfloor\ell\rfloor} \cdot \delta .
$$

Recall that $\delta \geq \frac{1}{\operatorname{poly}(n)}$. Thus there exists a constant $c$ such that if $\ell \geq c \log _{1+\varepsilon} n$, then the $\delta$-unit removal of water will decrease the potential by at least $2 n$. This forces $\phi(t)<\phi(t-1)$.

If, on the other hand, $\ell \leq c \log _{1+\varepsilon} n$, then the total potential $\phi(t)$ at the end of step $t$ can be at most

$$
n \int_{x=0}^{\ell}(1+\varepsilon)^{\lceil x\rceil} d x \leq \operatorname{poly}(n) .
$$

In both Case (1) and Case (2), at the end of each step $t$, the total potential $\phi(t)$ is either bounded by $\operatorname{poly}(n)$, or satisfies $\phi(t) \leq$ $\phi(t-1)$. It follows that the potential $\phi(t)$ is always bounded by $\operatorname{poly}(n)$, thereby completing the proof.

\section{A RANDOMIZED ALGORITHM FOR THE MULTI-PROCESSOR CUP GAME.}

In this section, we present a randomized algorithm for the multiprocessor cup game. Our algorithm achieves constant backlog with probability at least $1-2^{-\Omega\left(\varepsilon^{2} p\right)}$. In particular, when $\varepsilon$ is constant, this brings the probability of super-constant backlog down to exponentially small in $p$. In contrast, in the single-processor case, no bounds on constant backlog can be achieved with better than constant probability.

The main result of this section is:

Theorem 3. There exists a constant $c>0$ such that if $1 / 2 \geq \varepsilon \geq$ $p^{-1 / 3}$ and $2^{-c \varepsilon^{2} p}<\delta<1$, then there exists an algorithm for the multi-processor cup game that guarantees, after each step, a backlog of at most 3 with probability at least $1-2^{-\Omega\left(\varepsilon^{2} p\right)}$.

In the rest of the section, we motivate and define the algorithm used in Theorem 3 and give an overview of the algorithm's analysis; the full proof appears in [10].

For algebraic convenience, we consider a slight modification to the role that $\delta$ and $\varepsilon$ play: specifically, at each step the filler distributes $(1-\varepsilon) p$ units of water among the cups, placing no more than one unit in any cup (rather than $1-\delta$ ); and the emptier then selects up to $p+1$ (rather than $p$ ) distinct cups and removes up to $1+2 \delta$ from each (rather than 1 ). We call the multi-processor cup game with these modified resource-augmentation parameters the renormalized multi-processor cup game. Although the renormalized multi-processor cup game is not simply a rescaling of the multi-processor cup game, theorems about the renormalized multiprocessor cup game can nonetheless be reinterpreted as theorems about the multi-processor cup game; this is explained in detail in the extended paper [10].

The power of many players. We begin by presenting intuition for why having a large number of players might enable a backlog of $O(1)$. Consider a game in which, at each step, the filler distributes $(1-\varepsilon) p$ units of water, placing no more than one unit in any cup; and the emptier then selects $p$ cups to remove one unit of water from. (Notice that, for now, $\delta=0$.) Suppose the emptier follows the smoothed greedy algorithm. Since the number of integer thresholds crossed within each step of the smoothed greedy algorithm is a sum of independent $0-1$ variables, whose total mean is $(1-\varepsilon) p$, the probability of more than $p$ thresholds being crossed in a single step is at most $e^{-\varepsilon^{2} p / 6}$ by Chernoff bounds. Moreover, if a given $t$ has the property that each step $1,2, \ldots, t$ has individually crossed $p$ or fewer integer thresholds, then the emptier is able to maintain an integer fill of 0 at the end of each of the steps. By a union bound over the steps $1, \ldots, t$, it follows that, with probability at least $1-2^{-\Omega\left(\varepsilon^{2} p\right)}$, the backlog after each of the first $2^{\Omega\left(\varepsilon^{2} p\right)}$ steps is at most 1 . 
The difficulty of unbounded game lengths. Analyzing the smoothed greedy algorithm becomes more difficult when we consider games of longer length. When the filler crosses more than $p$ integer thresholds during a given step, we call the threshold crossings after the first $p$ surplus crossings. Surplus crossings force the emptier to try to catch up on later steps. However, a cup that is not fixed at step $t$ because of surplus crossings might have another threshold crossing at step $t+1$; since the emptier can undo at most one threshold crossing per cup per step, the emptier is then unable to fully catch up during step $t+1$. More generally, even when the integer fill of the system is large, if it is distributed over fewer than $p$ distinct cups, then the emptier is not be able to reduce the integer fill by a full $p$, which limits the emptier's ability to catch up on undoing past surplus crossings.

Mitigating surplus crossings with resource augmentation. Giving the emptier part of its final resource augmentation, so that they can remove $1+\delta$ units of water from each cup (rather than the $1+2 \delta$ augmentation that will be used later in the section), helps the emptier catch up in the face of surplus crossings.

This augmentation also removes an important property of the algorithm, however, which is that the emptier's emptying decisions should not affect the fractional amount of water within each cup (i.e., the amount of water modulo 1).

To handle this issue, we redefine the notion of an integer threshold as follows: if the total amount of water placed into cup $j$ by the filler in the first $t$ steps is denoted by $a_{j}(t)$, then cup $j$ crosses an integer threshold in step $t$ if and only if $\left\lfloor r_{j}+a_{j}(t)\right\rfloor>\left\lfloor r_{j}+a_{j}(t-1)\right\rfloor$. For each cup $j$, maintain a counter $w_{j}$ denoting the number of integer thresholds currently crossed. The counter $w_{j}$ is incremented whenever an integer threshold is crossed and is reduced to $\max \left(w_{j}-(1+\delta), 0\right)$ whenever water is removed from the cup. Given the new definition of integer thresholds, the new emptying algorithm removes water from the $p$ cups with largest counters $w_{j}$, removing $\min \left(w_{j}, 1+\delta\right)$ units of water from each. (This differs from Section 2, in which we ignore cups with less than 1 unit of water.)

The updated definition of integer thresholds allows for the emptier to make progress $1+\delta$ at reducing the number of integer thresholds in a given cup on a given step. Additionally, for simplicity of bookkeeping, the emptier is permitted to remove water from up to $p+1$ cups at each step, rather than $p$. The ability to remove from a $(p+1)$-st cup, along with the $\delta$ resource augmentation, together ensure that if there are surplus integer thresholds to catch up on during a given step (i.e., some counter $w_{j}$ is non-zero at the beginning of the step), the emptier makes at least $\delta$ progress towards catching up (i.e., the sum of the counters is at least $\delta$ smaller at the end of the step than at the start).

A new goal: bounding the surplus crossings. Call $\ell>0$ a backlog witness if the number of surplus crossings in steps $t-\ell+$ $1, \ldots, t$ is greater than $\delta \ell / 2$. The key to analyzing the algorithm after step $t$ is to show that, with probability $1-2^{-\Omega\left(\varepsilon^{2} p\right)}$, no $\ell>0$ are backlog witnesses. In particular, the emptier's algorithm is designed to make progress roughly $\delta$ at each step towards undoing any damage caused by past surplus crossings; if no $\ell>0$ are backlog witnesses, then we are guaranteed that after step $t$ the emptier has successfully handled all surplus thresholds from the past (i.e., the sum of the counters $w_{j}$ is 0 at the end of step $t$ ).

Whereas threshold crossings are independent between cups, surplus crossings require that at least $p+1$ cups all have thresholds crossed simultaneously. The lack of independence makes proving a concentration inequality potentially difficult. This prompts us to make one further modification to the algorithm.

Modifying the algorithm to make far-away crossings within a cup independent. Consider the following (re)definition of threshold crossings. Define uniformly random thresholds $r_{j}(i) \in$ $[i, i+1)$ for each cup $j$ and each $i \in 0,1,2, \ldots$, and then say that cup $j$ crosses a threshold at step $t$ whenever $a_{j}(t-1)<r_{j}(i) \leq a_{j}(t)$ for some $i$. This definition of threshold crossings adds independence between successive threshold crossings in a given cup but has one major issue: the filler could cause a single cup $j$ to cross two thresholds in the same step; this increases the counter $w_{j}$ by 2 , which causes the emptier to fall behind because they are only able to decrease the counter by $1+\delta$.

To resolve this issue, our actual algorithm uses a different layout of thresholds in which no two thresholds are within less than one of each other, while still maintaining independence between far-away thresholds. We assume w.l.o.g. that $1 / \delta \in \mathbb{N}$. Set the threshold $r_{j}(0)$ to be null in each cup $j$ (i.e., nonexistent). For every $m \in \mathbb{N}$ such that $m \equiv 1 \bmod (1+1 / \delta)$, select a random value $s_{j}(m) \in[0,1)$, and set

$$
r_{j}(m+k)= \begin{cases}\text { null, } & k=0, \\ m+k+s_{j}(m), & 0<k \leq 1 / \delta .\end{cases}
$$

We say that cup $j$ crosses a threshold at step $t$ whenever there is some non-null $r_{j}(i)$ for which $a_{j}(t-1)<r_{j}(i) \leq a_{j}(t)$.

The assignment of thresholds ensures that each step crosses at most one threshold in each cup, while also limiting to at most $1 / \delta$ the number of successive threshold crossings (in a given cup) that depend on the same random bits (i.e., on a given $s_{j}(i)$ ). The gaps that are inserted every $1 / \delta$ thresholds mean that only $1 / \delta$ thresholds are crossed for every $1 / \delta+1$ units of water poured into each cup. This discrepancy can be handled by giving the emptier a small amount of additional resource augmentation, allowing them to remove $1+2 \delta$ units of water from each cup, rather than just $1+\delta$.

The final algorithm works as follows: for every cup $j$, the algorithm maintains a threshold counter $w_{j}$, initially set to 0 , which is incremented by $1+\delta$ every time the cup crosses a threshold. The counter is reduced by the emptier (but never below 0 ) as follows: whenever the emptier empties from a cup $j$, they set $w_{j}=\max \left(w_{j}-(1+2 \delta), 0\right)$. When selecting which cups to empty from, the emptier selects arbitrarily from cups $j$ for which $w_{j} \geq 1+2 \delta$, and, if there are fewer than $p+1$ such cups, chooses the remaining cups (for which $w_{j}<1+2 \delta$ ) by maximum $w_{j}$.

Analyzing the algorithm. Recall that, in order to prove that the emptier keeps up with the filler and maintains a constant backlog, our goal is to bound the probability of there existing a backlog witness $\ell>0$ by $2^{-\Omega\left(\varepsilon^{2} p\right)}$.

Let $0<g<1$ be a sufficiently small constant. The case of $\ell \leq 2^{g \varepsilon^{2} p}$ is easily handled as a special case. In particular, by a union 
bound over the $2^{g \varepsilon^{2}} p$ steps leading up to step $t$, none of them have any surplus crossings (with probability at least $2^{g \varepsilon^{2}} p 2^{-\Omega\left(\varepsilon^{2} p\right)}=$ $2^{-\Omega\left(\varepsilon^{2} p\right)}$ and thus no $\ell \leq 2^{g \varepsilon^{2} p}$ is a backlog witness.

The difficulty in analyzing the algorithm is therefore to bound the probability of any $\ell>2^{g \varepsilon^{2}} p$ being a backlog witness. Define $T_{t}$ to be the number of surplus thresholds crossed during a step $t$. We complete the proof by demonstrating strong concentration bounds on the number $\sum_{m=t-\ell+1}^{t} T_{m}$ of surplus crossings within a sequence of $\ell$ steps. Specifically, we wish to show that, with probability $1-2^{-\Omega\left(\varepsilon^{2} p\right)}$, we have $\sum_{m=t-\ell+1}^{t} T_{m} \leq \delta \ell / 2$ for all $\ell>2^{g \varepsilon^{2}} p$, thereby prohibiting any $\ell>2^{g \varepsilon^{2}} p$ from being a backlog witness.

To prove a concentration bound, we use McDiarmid's inequality (i.e., Azuma's inequality applied to Doob Martingales) [2, 30]. McDiarmid's inequality says that if a random variable $Y$ can be expressed as a function $F\left(X_{1}, \ldots, X_{k}\right)$ of independent random variables $X_{1}, \ldots X_{k}$, and if each $X_{j}$ 's value can affect $F\left(X_{1}, \ldots, X_{k}\right)$ by at most some bounded amount $c$, then a Chernoff-style concentration bound holds for $Y$ :

$$
\operatorname{Pr}[Y \geq \mathbb{E}[Y]+R] \leq e^{-2 R^{2} /\left(c^{2} k\right)} .
$$

Define a threshold collection with random value $s_{j}(i)$ to be the set of thresholds $r_{j}(i)$ whose random offset modulo 1 is determined by $s_{j}(i)$. Define the hitting capacity of a threshold collection $H$ in a cup $j$ to be the expected number of threshold in $H$ to be crossed during steps $t-\ell+1, \ldots, t$. (Note that the hitting capacity is a function only of how much water the filler places into cup $j$ at each step, and not a function of the emptier's behavior.)

A first attempt at applying McDiarmid's inequality might go as follows. Define $Y=\sum_{m=t-\ell+1}^{t} T_{m}$ to be the number of surplus crossings in the $\ell$ steps leading up to and including $t$. Notice that $Y$ can be written as a function $F$ of the random values $s_{j}(i)$ of each of the threshold collections with non-zero hitting capacities. As a loose bound, there are at most $n+\ell p$ threshold collections with nonzero hitting capacities, since in order for a cup $j$ to have $h>1$ such threshold collections, at least $(h-1)$ units of water must have been poured into the cup during the steps $t-\ell+1, \ldots, t$ (that way the water could cross the threshold-less gap between the collections). Moreover, the random value of each threshold collection can affect the number of surplus crossings $Y$ addititively by at most $1 / \delta$, since the random value $s_{j}(i)$ controls when at most $1 / \delta$ different threshold crossings occur. Thus, we can apply McDiarmid's inequality with $c=1 / \delta$ and $k=n+\ell p$.

Since $\mathbb{E}[Y]$ is small relative to $\delta \ell / 2$, we wish to apply McDiarmid's inequality with $R \approx \delta \ell / 2$. In order for McDiarmid's inequality to be useful, we need for $\frac{R^{2}}{c^{2} k}$ to be large. Expanding the quantity gives,

$$
\frac{R^{2}}{c^{2} k}=\frac{\delta^{2} \ell^{2} / 4}{\delta^{-2}(n+\ell p)}
$$

If $n$ is large relative to $\ell^{2}$, then we run into a problem, which is that the number $k$ of random variables to which we are applying McDiarmid's inequality is too large for McDiarmid's inequality to be useful. If, on the other hand, $n$ is small (say, $n \leq \ell p$ ), then

$$
\frac{R^{2}}{c^{2} k}=\Theta\left(\frac{\ell}{\delta^{-4} p}\right) \geq \Omega(\sqrt{\ell}),
$$

since $\ell>2^{-g \varepsilon^{2} p}$ dominates $\delta^{-4} p$. (Recall that $\delta^{-1}$ is assumed by Theorem 3 to be sufficiently small in $2^{O\left(\varepsilon^{2} p\right)}$.) Thus if we assume that $n \leq \ell p$, then McDiarmid's inequality bounds the probability of $\ell$ being a backlog witness by $2^{-\Omega(\sqrt{\ell})}$ for each $\ell>2^{g \varepsilon^{2}} p$; this probability is small enough that a union bound over all $\ell \geq 2^{g \varepsilon^{2}} p$ completes the proof of the theorem.

To handle the case of $\ell p \ll n$, we modify our application of McDiarmid's inequality in order to reduce the number of random variables $k$ to $O(\ell p)$, without significantly increasing $c$. Specifically, we express $Y$ as a function of random variables $X_{1}, \ldots, X_{p \ell+1}$ where each $X_{s}$ corresponds with the random values $s_{j}(i)$ of some set of threshold collections, rather than with just a single random value. That is, we partition the threshold collections into components $Q_{1}, \ldots, Q_{p \ell+1}$, and then define for each component $Q_{s}$ a random variable $X_{s}$ that contains the random values of the threshold collections in the component $Q_{s}$. The key is to design the partition $Q_{1}, \ldots, Q_{p \ell+1}$ so that McDiarmid's inequality can subsequently be applied. This is accomplished by assigning threshold collections that are likely to have substantial effect on $Y$ to their own components, and then grouping together large sets of threshold collections that are not expected to have substantial effect on $Y$ into other components. Formally, this is accomplished by assigning threshold collections with hitting capacity 1 or greater to their own partition components, and then grouping together threshold components with hitting capacities less than 1 so that the sum of the hitting capacities within each partition component is between 1 and 2. We show that with high probability in $\ell p$, no individual $X_{s}$ has substantial effect on the outcome of $Y$ (i.e., an additive effect of more than $\left.O\left(\delta^{-1}+\log (\ell p)\right)\right)$, allowing us to obtain the desired concentration bound via McDiarmid's inequality. The full argument appears in the extended paper [10].

Bounding the probability of super-constant backlog. We conclude the section by extending the algorithm analysis to the probability of arbitrary backlogs occurring. In the algorithm described above, it there are multiple cups containing super-constant amounts of water (i.e., cups for which $w_{j} \geq 1+2 \delta$ ), the emptier may select which of them to empty out of arbitrarily. We define the multiprocessor smoothed greedy algorithm to be the strategy in which, when deciding between cups of equal priority, the emptier always chooses the fullest cups.

Theorem 4. There exists a constant $c>0$ such that if $1 / 2 \geq \varepsilon \geq$ $p^{-1 / 3}, \delta \geq \frac{1}{\operatorname{poly}(p)}$, and $2^{-c \varepsilon^{2} p}<\delta<1$, the multi-processor smoothed greedy algorithm on $n$ cups for the (renormalized) multi-processor cup game has the following guarantee: for any value $k \geq \log p$ and for any step $t$, the backlog is $O(k / \varepsilon)$ after step $t$ with probability at least $1-O\left((1 / 2)^{2^{k}}\right)$.

The analysis combines ideas from each of the previous sections. We consider a dynamic version of the multi-processor cup game being played on the cups containing $4+\delta$ or more units of water. The number of cups involved in the game at the end of a step $t$ is upper bounded by the number of surplus thresholds that have been crossed in the past without being subsequently accounted for by the emptier. Concentration bounds for the number of cups in the dynamic game can thus be proven using extensions of the ideas 
used to prove Theorem 3. Combining this with an analysis of the deterministic greedy algorithm for the dynamic multi-processor cup game, we obtain the desired bound on backlog.

\section{LOWER BOUNDS}

In this section we discuss strategies that the filler can follow in order to maximize backlog, regardless of the algorithm followed by the emptier. When $\varepsilon$ is a positive constant, the resulting bounds establish that the algorithms for the emptier given in the preceding sections are optimal up to constant-factor changes in the backlog and in $p$.

Consider a cup-emptying game on $n$ cups in which at each step, the filler is allowed to distribute $p / 2$ units of water among the cups, and then the emptier is allowed to select $p$ cups and remove all of the water from each of them. We call this the universal emptying game on $p$ processors and $n$ cups. Lower bounds for the universal emptying game easily port to lower bounds for the single-processor cup game and the multi-processor cup game (played with arbitrary $\varepsilon$ for the single-processor variant, and with arbitrary $\varepsilon \leq 1 / 2$ and $\delta$ for the multi-processor variant).

We begin with a lower bound for the universal emptying game when the filler is an adaptive adversary.

Theorem 5. Consider the universal emptying game on $p$ processors and $n$ cups, where $n$ is a multiple of $p$. There is an adaptive strategy for the filler that accomplishes a backlog of $\Theta\left(\log \frac{n}{p}\right)$ after step $\frac{n}{p}-1$.

Proof. The strategy that the filler follows is a simple variant on the strategy used by past authors in the single-processor cup game $[12,18]$. At the beginning of each step $t$, the emptier has so far removed water from (up to) $p(t-1)$ cups. The filler ignores these cups, and distributes $p / 2$ units of water among the $n-p(t-1)$ cups that have never had water removed from them. This continues until the end of the $\frac{n}{p}$-th step, at which point every cup has been emptied.

After the $t$-th step, there are $n-p t$ cups each containing

$$
\frac{p / 2}{n}+\frac{p / 2}{n-p}+\frac{p / 2}{n-2 p}+\cdots+\frac{p / 2}{n-p(t-1)}
$$

water. It follows that at the end of step $\frac{n}{p}-1$ some cup has fill at least

$$
\frac{p}{2} \cdot\left(\frac{1}{n}+\frac{1}{n-p}+\cdots+\frac{1}{2 p}\right)=\sum_{j=2}^{n / p} \frac{1}{2 j}=\Theta\left(\log \frac{n}{p}\right) .
$$

Theorem 5 implies that our backlog upper bound of $O\left(\frac{1}{\varepsilon} \log n\right)$ (given by Theorem 2) for the multi-processor cup game is optimal (up to constant factors in the backlog) when $\varepsilon \leq 1 / 2$ is a positive constant and $n \geq p^{2}$. This is captured formally in the following corollary.

Corollary 2. For $n \geq p^{2}$, any deterministic emptying strategy for the multi-processor cup game played on $n$ cups must have worst-case backlog at least $\Omega(\log n)$.
Next, we generalize Theorem 5 to the case where the filler is an oblivious adversary, meaning the filler is unable to see which cups the emptier has removed water from.

Theorem 6. Consider the universal emptying game on $p$ processors and $n$ cups. For any $k$ such that $2 p \leq k \leq n$ and such that $k$ is a multiple of $p$, there is an oblivious strategy for the filler that accomplishes a backlog of $\Theta\left(\log \frac{k}{p}\right)$ after step $\frac{k}{p}-1$ with probability at least

$$
\left(\frac{1}{\left(\begin{array}{c}
k \\
p
\end{array}\right)}\right)^{k / p-1} \geq \frac{1}{k^{k}}
$$

Proof. In order to achieve a backlog of $\Theta\left(\log \frac{k}{p}\right)$, the filler considers only the cups $1, \ldots, k$ and attempts to follow the same construction as presented in the proof of Theorem 5 . The caveat is that the filler must now guess during each step which $p$ (or fewer) cups the emptier selected in the previous step. Since the filler is concerned only about the first $k$ cups, it suffices for the filler to guess after each step $t$ a $p$-element subset $S_{t} \subseteq[k]$ such that during the $t$-th step, $S_{t}$ contains the cups in $[k]$ out of which the emptier emptied. The number of options for each $S_{t}$ is $\left(\begin{array}{l}k \\ p\end{array}\right)$. Thus the filler can successfully simulate the adaptive lower-bound construction on $k$ cups with probability at least

$$
\left(\frac{1}{\left(\begin{array}{c}
k \\
p
\end{array}\right)}\right)^{k / p-1} \geq\left(\frac{1}{k^{p}}\right)^{k / p-1} \geq \frac{1}{k^{k}} .
$$

Applying Theorem 6 with the appropriate choices of parameters yields two interesting corollaries. Corollary 3 implies that Theorems 1 and 4, which upper bound the backlog of the smoothed greedy algorithm and the multi-processor smoothed greedy algorithm, are optimal (up to constant factors in the backlog and $p$ ) when $\varepsilon \leq 1 / 2$ is a positive constant.

Corollary 3. Consider the p-processor multi-processor cup game on $n$ cups with $\varepsilon \leq 1 / 2$. For any $k$ satisfying $\log p \leq k \leq \log n$, there is an oblivious pouring strategy that guarantees after after some step a backlog of at least $\Omega(k)$ with probability at least $2^{-2^{k}}$.

Corollary 4 ensures that Theorem 3, which ensures in the multiprocessor cup game a backlog of three or smaller with probability at least $1-e^{-\Omega\left(\varepsilon^{2} p\right)}$, is optimal (up to constant factors in $p$ ) when $\varepsilon \leq 1 / 2$ is a positive constant. This is captured formally in the following corollary.

Corollary 4. Consider the p-processor multi-processor cup game with $\varepsilon \leq 1 / 2$. For any constant $c$, there is an oblivious pouring strategy that guarantees after at some step a backlog of at least $c$ with probability at least $2^{-O(p)}$.

\section{RECOVERY FROM BAD STARTING STATES}

We now revisit the single-processor cup game and the multiprocessor cup game when the starting state of the cups is nonempty. In particular, suppose that $b$ units of water have already been dispersed among the cups arbitrarily before the game begins. 
Theorems 7 and 8 establish that in both games the system can quickly recover from such a starting state.

Theorem 7. Consider the single-processor cup game beginning from a starting state in which $b$ units of water have already been dispersed arbitrarily among the cups. Suppose $\varepsilon \leq 1 / 2$ and consider some value $k \geq 0$ such that $3 \log \frac{1}{\varepsilon} \leq k$. Then for $t>\frac{b}{\varepsilon}$, the probability that the smoothed greedy algorithm has backlog $k$ or greater after step $t$ is at $\operatorname{most}(1 / 2)^{2^{\Omega(k)}}$.

Theorem 8. Consider the (renormalized) multi-processor cup game beginning from a starting state in which $b$ units of water have already been dispersed arbitrarily among cups. Set each counter $w_{j}(0)$ in the multi-processor smoothed greedy algorithm initially to be the number of thresholds crossed by the $b$ units of water in each cup $j$.

There exists a constant $c>0$ such that if $1 / 2 \geq \varepsilon \geq p^{-1 / 3}$ and $2^{-c \varepsilon^{2} p}<\delta<1$, then the following holds.

The multi-processor smoothed greedy algorithm guarantees after any given step $t$ satisfying $t \geq \delta^{-8} p^{2}$ and $t \geq 2 b / \delta$, that

- The backlog is three or less with probability at least

$$
1-e^{-\Omega\left(\varepsilon^{2} p\right)}-e^{-\Omega\left(t^{1 / 6}\right)} .
$$

- Assuming that $\delta \geq 1 / \operatorname{poly}(p)$, then for any $k \geq \log p$, the backlog is $O(k / \varepsilon)$ with probability at least

$$
1-O\left(e^{-e^{k}}\right)-e^{-\Omega\left(t^{1 / 6}\right)} .
$$

The proofs of Theorems 7 and 8 rely on simple extensions of the ideas used to prove Theorems 1, 3, and 4. In each case, the resource augmentation given to the emptier allows for them eventually to compensate for the initial bad starting state. The full proofs of Theorems 7 and 8 are deferred to the full version of the paper [10].

\section{REFERENCES}

[1] Micah Adler, Petra Berenbrink, Tom Friedetzky, Leslie Ann Goldberg, Paul Goldberg, and Mike Paterson. 2003. A Proportionate Fair Scheduling Rule with Good Worst-case Performance. In Proceedings of the Fifteenth Annual ACM Symposium on Parallel Algorithms and Architectures (SPAA). 101-108. https://doi.org/10.1145/777412.777430

[2] Noga Alon and Joel H Spencer. 2004. The probabilistic method. John Wiley \& Sons.

[3] Amihood Amir, Martin Farach, Ramana M. Idury, Johannes A. La Poutré, and Alejandro A. Schäffer. 1995. Improved Dynamic Dictionary Matching. Inf. Comput. 119, 2 (1995), 258-282. https://doi.org/10.1006/inco.1995.1090

[4] Amihood Amir, Gianni Franceschini, Roberto Grossi, Tsvi Kopelowitz, Moshe Lewenstein, and Noa Lewenstein. 2014. Managing unbounded-length keys in comparison-driven data structures with applications to online indexing. SIAM 7 . Comput. 43, 4 (2014), 1396-1416.

[5] Yossi Azar and Arik Litichevskey. 2006. Maximizing throughput in multi-queue switches. Algorithmica 45, 1 (2006), 69-90.

[6] Amotz Bar-Noy, Ari Freund, Shimon Landa, and Joseph (Seffi) Naor. 2002 Competitive On-line Switching Policies. In Proceedings of the Thirteenth Annual ACM-SIAM Symposium on Discrete Algorithms (SODA). 525-534. http: //dl.acm.org/citation.cfm?id=545381.545452

[7] Amotz Bar-Noy, Aviv Nisgav, and Boaz Patt-Shamir. 2002. Nearly optimal perfectly periodic schedules. Distributed Computing 15, 4 (2002), 207-220.

[8] S. K. Baruah, N. K. Cohen, C. G. Plaxton, and D. A. Varvel. 1996. Proportionate progress: A notion of fairness in resource allocation. Algorithmica 15, 6 (01 Jun 1996), 600-625. https://doi.org/10.1007/BF01940883

[9] Sanjoy K Baruah, Johannes E Gehrke, and C Greg Plaxton. 1995. Fast scheduling of periodic tasks on multiple resources. In Proceedings of the 9th International Parallel Processing Symposium. 280-288.

[10] Michael Bender, Martín Farach-Colton, and William Kuszmaul. 2019. Achieving Optimal Backlog in Multi-Processor Cup Games. arXiv preprint arXiv:1904.02861 (2019).
[11] Michael A Bender, Jake Christensen, Alex Conway, Martín Farach-Colton, Rob Johnson, and Meng-Tsung Tsai. 2018. Optimal Ball Recycling. In Proceedings of the Symposium on Discrete Algorithms (SODA). 2527-2546.

[12] Michael A. Bender, Sándor P. Fekete, Alexander Kröller, Vincenzo Liberatore, Joseph S. B. Mitchell, Valentin Polishchuk, and Jukka Suomela. 2015. The minimum backlog problem. Theor. Comput. Sci. 605 (2015), 51-61. https: //doi.org/10.1016/j.tcs.2015.08.027

[13] Marijke Hans L. Bodlaender, Cor A. J. Hurkens, Vincent J. J. Kusters, Frank Staals, Gerhard J. Woeginger, and Hans Zantema. 2012. Cinderella versus the Wicked Stepmother. In IFIP International Conference on Theoretical Computer Science. 57-71.

[14] Marijke H. L. Bodlaender, Cor A. J. Hurkens, and Gerhard J. Woeginger. 2011. The Cinderella Game on Holes and Anti-holes. In Proceedings of the 37th International Conference on Graph-Theoretic Concepts in Computer Science (WG). 71-82. https: //doi.org/10.1007/978-3-642-25870-1 8

[15] Marek Chrobak, János Csirik, Csanád Imreh, John Noga, Jirí Sgall, and Gerhard J. Woeginger. 2001. The Buffer Minimization Problem for Multiprocessor Scheduling with Conflicts. In Proceedings of the 28th International Colloquium on Automata, Languages and Programming (ICALP), Vol. 2076. 862-874. https://doi.org/10.1007/3-540-48224-5_70

[16] Peter Damaschke and Zhen Zhou. 2005. On queuing lengths in on-line switching. Theoretical computer science 339, 2-3 (2005), 333-343.

[17] Paul Dietz and Daniel Sleator. 1987. Two Algorithms for Maintaining Order in a List. In Proceedings of the Nineteenth Annual ACM Symposium on Theory of Computing (STOC). 365-372. https://doi.org/10.1145/28395.28434

[18] Paul F. Dietz and Rajeev Raman. 1991. Persistence, Amortization and Randomization. In Proceedings of the Second Annual ACM-SIAM Symposium on Discrete Algorithms (SODA). 78-88. http://dl.acm.org/citation.cfm?id=127787.127809

[19] Johannes Fischer and Paweł Gawrychowski. 2015. Alphabet-dependent string searching with wexponential search trees. In Annual Symposium on Combinatorial Pattern Matching (CPM). 160-171.

[20] Rudolf Fleischer and Hisashi Koga. 2004. Balanced Scheduling toward Loss-Free Packet Queuing and Delay Fairness. Algorithmica 38, 2 (01 Feb 2004), 363-376. https://doi.org/10.1007/s00453-003-1064-z

[21] H Richard Gail, G Grover, Roch Guérin, Sidney L Hantler, Zvi Rosberg, and Moshe Sidi. 1993. Buffer size requirements under longest queue first. Performance Evaluation 18, 2 (1993), 133-140.

[22] Leszek Gasieniec, Ralf Klasing, Christos Levcopoulos, Andrzej Lingas, Jie Min, and Tomasz Radzik. 2017. Bamboo Garden Trimming Problem (Perpetual maintenance of machines with different attendance urgency factors). In International Conference on Current Trends in Theory and Practice of Informatics. Springer, 229-240.

[23] Michael H. Goldwasser. 2010. A survey of buffer management policies for packet switches. SIGACT News 41, 1 (2010), 100-128. https://doi.org/10.1145/1753171. 1753195

[24] Michael T Goodrich and Paweł Pszona. 2013. Streamed graph drawing and the file maintenance problem. In International Symposium on Graph Drawing. Springer, 256-267.

[25] Tsvi Kopelowitz. 2012. On-line indexing for general alphabets via predecessor queries on subsets of an ordered list. In Proceedings of the 53rd Annual Symposium on Foundations of Computer Science (FOCS). 283-292.

[26] Ami Litman and Shiri Moran-Schein. 2005. On distributed smooth scheduling. In Proceedings of the Seventeenth Annual ACM Symposium on Parallelism in Algorithms and Architectures (SPAA). 76-85.

[27] Ami Litman and Shiri Moran-Schein. 2009. Smooth Scheduling Under Variable Rates or the Analog-Digital Confinement Game. Theor. Comp. Sys. 45, 2 (June 2009), 325-354. https://doi.org/10.1007/s00224-008-9134-x

[28] Ami Litman and Shiri Moran-Schein. 2011. On centralized smooth scheduling. Algorithmica 60, 2 (2011), 464-480.

[29] Chung Laung Liu. 1969. Scheduling algorithms for multiprocessors in a hard real-time environment. FPL Space Programs Summary, 1969 (1969).

[30] Colin McDiarmid. 1989. On the method of bounded differences. Surveys in combinatorics 141, 1 (1989), 148-188.

[31] Mark Moir and Srikanth Ramamurthy. 1999. Pfair scheduling of fixed and migrating periodic tasks on multiple resources. In Proceedings of the 20th IEEE Real-Time Systems Symposium. 294-303.

[32] Christian Worm Mortensen. 2003. Fully-dynamic two dimensional orthogonal range and line segment intersection reporting in logarithmic time. In Proceedings of the Fourteenth Annual ACM-SIAM Symposium on Discrete Algorithms (SODA). 618-627.

[33] Michael Rosenblum, Michel X Goemans, and Vahid Tarokh. 2004. Universal bounds on buffer size for packetizing fluid policies in input queued, crossbar switches. In Twenty-third Annual Joint Conference of the IEEE Computer and Communications Societies (INFOCOM), Vol. 2. 1126-1134.

[34] Daniel D. Sleator and Robert E. Tarjan. 1985. Amortized efficiency of list update and paging rules. Commun. ACM 28, 2 (1985), 202-208. 